\title{
The Relationship between the Tissue Expression of TLR2, TLR4, TLR5, and TLR7 and Systemic Inflammatory Responses in Colorectal Cancer Patients
}

Beilmann-Lehtonen, Ines

2021-09-08

Beilmann-Lehtonen , I , Hagström , J , Kaprio , T , Stenman , U-H , Strigård , K , Palmqvist , R, Gunnarsson, U , Böckelman , C \& Haglund, C 2021, ' The Relationship between the Tissue Expression of TLR2, TLR4, TLR5, and TLR7 and Systemic Inflammatory Responses in Colorectal Cancer Patients ' , Oncology , vol. 99 , no. 12 , 10.1159/000518397 . https://doi.org/10.1159/00051839

http://hdl.handle.net/10138/338688

https://doi.org/10.1159/000518397

acceptedVersion

Downloaded from Helda, University of Helsinki institutional repository.

This is an electronic reprint of the original article.

This reprint may differ from the original in pagination and typographic detail.

Please cite the original version. 
The Relationship Between the Tissue Expression of TLR2, TLR4, TLR5, and TLR7 and Systemic Inflammatory Responses in Colorectal Cancer Patients

Ines Beilmann-Lehtonen ${ }^{1,2,5}$, Jaana Hagström²,3,4, Tuomas Kaprio ${ }^{5}$, Ulf-Håkan Stenman ${ }^{6}$, Karin Strigård $^{7}$, Richard Palmqvist ${ }^{8}$, Ulf Gunnarsson ${ }^{7}$, Camilla Böckelman ${ }^{2,5 *}$, Caj Haglund ${ }^{2,3,5 *}$

${ }^{1}$ Department of Transplantation and Liver Surgery, University of Helsinki and Helsinki University Hospital, Helsinki, Finland

${ }^{2}$ Translational Cancer Medicine Research Program, Faculty of Medicine, University of Helsinki, Helsinki, Finland

${ }^{3}$ Department of Pathology, University of Helsinki and Helsinki University Hospital, Helsinki, Finland ${ }^{4}$ Department of Oral Pathology and Radiology, University of Turku, Turku, Finland

${ }^{5}$ Department of Gastrointestinal Surgery, University of Helsinki and Helsinki University Hospital, Helsinki, Finland

${ }^{6}$ Department of Clinical Chemistry, University of Helsinki, Helsinki, Finland

${ }^{7}$ Department of Surgical and Perioperative Sciences, Surgery, Umeå University, Umeå, Sweden

${ }^{8}$ Department of Medical Biosciences, Pathology, Umeå University, Umeå, Sweden

${ }^{*}$ Equal contribution.

Running title: Evaluation of tissue TLRs and plasma CRP in colorectal cancer

Abbreviations: CI, confidence interval; CRC, colorectal cancer; CRP, C-reactive protein; DAMP, damage-associated molecular pattern; DC, dendritic cell; DSS, disease-specific survival; GPS, Glasgow prognostic score; HR, hazard ratio; IQR, interquartile range; LMR, lymphocyte-tomonocyte ratio; NLR, neutrophil-to-lymphocyte ratio; PAMP, pathogen-associated molecular pattern; PLR, platelet-to-lymphocyte ratio; SIR, systemic inflammatory response; TIMP-1, tissue inhibitor of metalloproteinase-1; TLR, toll-like receptor; TMA, tissue microarray; TR-IFMA, timeresolved immunofluorometric assay.

Correspondence to:

Ines Beilmann-Lehtonen, MD

Department of Transplantation and Liver Surgery, Helsinki University Hospital Haartmaninkatu 4

PO Box 340 
FIN-00029 HUS Finland

Phone: +358400293938

Email: ines.beilmann-lehtonen@hus.fi

Keywords: toll-like receptor, colorectal cancer, immunohistochemistry, survival, prognosis

\begin{abstract}
Background: Colorectal cancer (CRC) is the third most commonly diagnosed malignancy globally. CRC patients with elevated plasma C-reactive protein (CRP) levels exhibit compromised prognoses. Toll-like receptors (TLRs), activating the innate and adaptive immune system, may contribute to proand antitumorigenic inflammatory responses. We aimed to identify a possible link between local and systemic inflammatory responses in CRC patients by investigating the association between tissue TLRs and plasma CRP.
\end{abstract}

Methods: Tissue expressions of TLR2, TLR4, TLR5, and TLR7 were assessed using immunohistochemistry of tissue microarray (TMA) slides from 549 CRC patients surgically treated between 1998 and 2005. Blood samples were drawn preoperatively, centrifuged, aliquoted, and stored at $-80^{\circ} \mathrm{C}$ until analysis. Plasma CRP was determined through high-sensitivity time-resolved immunofluorometric assay (TR-IFMA). We investigated the association of TLRs to clinicopathological variables, plasma CRP, and survival.

Results: High TLR2 expression [hazard ratio (HR) 0.059; 95\% confidence interval (CI) 0.41-0.85; $p=0.005$ ], high TLR5 expression (HR 0.60; 95\% CI 0.45-0.83; $p=0.002$ ), positive TLR7 expression (HR 0.49; 95\% CI 0.33-0.72; $p<0.001$ ), and low CRP (HR 1.48; 95\% CI 1.08-2.11; $p=0.017$ ) associated to a better prognosis. A high TLR2 immunoexpression associated with a better prognosis among low CRP patients (HR 0.53; 95\% CI 0.35-0.80; $p=0.002$ ), high TLR4expression among high CRP patients (HR 2.04; 95\% CI 1.04-4.00; $p=0.038$ ), high TLR5 expression among low CRP patients (HR 0.059; 95\% CI 0.37-0.92; $p=0.021$ ), and positive TLR7 expression among low CRP 
patients (HR 0.53; 95\% CI 0.28-1.00; $p=0.049)$. In multivariate analyses, no biomarkers emerged as significant independent variables.

Conclusions: High tissue TLR2, TLR5, and TLR7 levels associate with a better prognosis. Among low CRP patients, those with high TLR2, TLR5, and TLR7 immunoexpressions exhibited a better prognosis. Among high CRP patients, a low TLR4 immunoexpression associated with a better prognosis.

\section{Introduction}

Colorectal cancer (CRC) is the second leading malignancy in terms of cancer deaths in the world, and third most common in terms of cancer incidence [1]. Although prognosis among CRC patients has improved, recurrence develops in $17 \%$ of stage II and $36 \%$ of stage III patients [2]. Reliable prognostic markers are needed to identify individuals at an increased risk who may benefit from tailored adjuvant therapies and intensified surveillance.

Chronic local inflammatory processes represent known risk factors for developing CRC [3]. A higher density of tumor-infiltrating lymphocyte (TIL), however, may promote antitumoral immune mechanisms and, thus, associate with better survival among CRC patients [4].

Transmembranous toll-like receptors (TLRs), expressed by innate immune system cells, Band T-lymphocytes, epithelial cells of the gastrointestinal tract and the respiratory system, are crucial to activating the host's innate immune responses after recognizing pathogen-associated molecular patterns (PAMPs) and endogenous damage-associated molecular patterns (DAMPs) [5-7]. In addition, TLRs form a part of the adaptive immune system by initiating dendritic cell (DC) maturation, migration to lymph nodes, and signaling to naive T-cells [8].

TLRs take part in the pathogenesis of several diseases and contribute to the development of malignancies [5,7]. In carcinogenesis, the role of TLRs vary since the same TLRs may act as tumorpromoting in some and antitumorigenic in other malignancies $[6,9,10]$. 
TLRs have not been extensively investigated in CRC, with TLR4 representing the most studied TLR in CRC. In a previous study, we revealed that Dukes B patients with strong TLR4 tissue immunoexpression exhibited a worse prognosis, while a strong TLR2 tissue immunoexpression associated with a favorable prognosis in Dukes C patients [11]. In another study, we demonstrated that a strong TLR5 tissue expression represents an independent positive prognostic factor among CRC patients [12]. To our knowledge, this was the first study to describe the role of TLR5 in CRC. In addition, a low serum TLR2 associated with a high serum C-reactive protein (CRP) in another published study [13].

Several studies have demonstrated that elevated levels of acute-phase protein CRP, a wellknown marker for the systemic inflammatory response (SIR), predicts a poorer prognosis in CRC patients [14-17]. A high Glasgow prognostic score (GPS) or modified GPS (mGPS), based on CRP and albumin levels, predict a worse prognosis in CRC patients [18].

The relationship between the local and systemic inflammatory responses in CRC was previously investigated in some studies. A low CD4+ T-lymphocyte infiltration, a predictor of poorer survival, associated with elevated CRP levels in CRC patients [19]. In another study, a significant negative association was detected between a high CRP and intratumoral T-regulatory FOXP3+ cell infiltration [20]. Among stage II and III CRC patients with mild local inflammatory cell inflitration, prognosis appeared better in patients with a low mGPS score [21].

The underlying mechanisms connecting the local and systemic inflammatory responses remain unclear, with further studies needed. Thus, our study aimed to evaluate the possible relationship between local and systemic inflammatory responses in CRC by comparing the tissue immunoexpressions of TLR2, TLR4, TLR5, and TLR7 and plasma CRP values.

\section{Materials and Methods}

\section{Patients}


We retrospectively studied a cohort of 549 consecutive CRC patients surgically treated in the Department of Surgery, Helsinki University Hospital, Finland, between 1998 and 2005 (Table 1). The median age at surgery was 69.2 [interquartile range (IQR) 59.2-77.4], and 260 (47.4\%) patients were women. The median follow-up time was 6.44 years (IQR 2.00-14.84). By the end of follow-up, $170(31.0 \%)$ patients were alive and $191(34.9 \%)$ patients had died of CRC. The 5-year diseasespecific survival (DSS) for all patients was 69.2\% [95\% confidence interval (CI) 65.1-63.3]. We used the sixth version of the TNM disease classification [22] for CRC staging, summarized in Table 1. The clinical data were gathered from patient medical records, while survival data and the cause of death of deceased patients were provided by the Population Register Center of Finland (currently, Digital and Population Data Service Agency) and Statistics Finland.

The Surgical Ethics Committee of Helsinki University Hospital (Dnro HUS 226/E6/06, extension TMK02 $§ 66$ 17.4.2013) approved the study protocol. The National Supervisory Authority of Health and Welfare (Valvira Dnro 10041/06.01.03.01/2012) permitted the use of archival tissue samples and blood samples without requiring individual consent.

\section{Blood samples}

Patient blood samples were retrieved before surgery (range 0-30 days), in most cases within 3 days of surgery $(92.7 \%)$. After centrifuging, serum and plasma components were stored as aliquoted at $80^{\circ} \mathrm{C}$ until analysis. Plasma CRP was determined through a high-sensitivity method [time-resolved immunofluorometric assay (TR-IFMA) using a monocolonal CRP antibody (anti-hCRP, code 6405, Medix Biochemica, Espoo, Finland)] as described elsewhere [23].

\section{Tissue samples}

Formalin-fixed and paraffin-embedded surgical tumor samples were stored in the archives of the Department of Pathology, Helsinki University Hospital. Representative tumor areas were marked on 
hematoxylin- and eosin-stained slides by an experienced pathologist (JH). Four 1.0-mm cores were punched from these representative tumor areas and the tissue microarray (TMA) blocks were constructed using a TMA Grand Master 3D instrument (Histech Ltd Budapest, Hungary). For immunohistochemistry, we cut 4- $\mu$ m sections from the TMA blocks as previously described [24].

\section{Immunohistochemistry}

We used the same immunohistochemical staining protocol for each TLR. The 4- $\mu \mathrm{m}$ TMA sections were deparaffinized and rehydrated. For antigen retrieval, the slides were prewarmed in a PreTreatment module (Lab Vision UK Ltd, UK) and treated with a Tris-HCl buffer ( $\mathrm{pH}$ 9) for 15 min at $98^{\circ} \mathrm{C}$. We used the Autostainer 480 (Lab Vision, Fremont, California, USA), with the REAL EnVision Detection System (peroxidase/DAB+, rabbit/mouse; Dako, Glostrup, Denmark), for staining the TMA slides. The endogenous peroxidases were blocked with a $0.3 \%$ Dako REAL Peroxidase-Blocking Solution incubation for $5 \mathrm{~min}$, followed by primary antibody incubation with primary antibodies: $200 \mu \mathrm{g} / \mathrm{ml}$ TLR2 rabbit polyclonal (sc-10739, Santa Cruz Biotechnology, Santa Cruz, CA, USA; diluted to 1:200; overnight), $200 \mu \mathrm{g} / \mathrm{ml}$ TLR4 mouse monoclonal (sc-293072, Santa Cruz Biotechnology, Santa Cruz, CA, USA; diluted to $1: 2000 ; 1 \mathrm{hr}), 1.0 \mu \mathrm{g} / \mathrm{ml}$ TLR5 mouse monoclonal (NBP2-24787, Novus Biologicals, Centennial, USA; diluted to 1:300; overnight), and $1.0 \mu \mathrm{g} / \mathrm{ml}$ TLR7 rabbit polyclonal (NBP2-24906, Novus Biologicals, Centennial, USA; diluted to $1: 300 ; 1 \mathrm{hr})$. Finally, we incubated for $30 \mathrm{~min}$ with the peroxidase-conjugated Dako REAL EnVision/HRP, Rabbit/Mouse (ENV) secondary antibody, and visualized the staining using Dako REAL DAB + Chromogen for 10 min. Slides were counterstained with Meyer's hematoxylin and mounted in Pertex Mounting (Histolab Products AB, Sweden). In each staining series, we used negative (specimens processed without primary antibody) and positive (tonsillar, skin, and cutaneous squamous cell carcinoma tissue, known to show a high immunoreactivity for the studied antigens) controls. 


\section{Scoring of samples}

Staining intensities of TLR2, TLR4, TLR5, and TLR7 in CRC TMAs were evaluated by two independent assessors (IB-L and $\mathrm{JH}$ ), one of whom is an experienced pathologist from the Department of Pathology, Helsinki University Hospital. Both assessors were blinded from the clinical data. The immunoreactivity of the TLRs was scored from 0 to 3 as follows: 0 as negative, 1 as weak positive, 2 as moderate, and 3 as a strong immunoreactivity (Fig. 1). TLR2 and TLR4 immunopositivity appeared as a cytoplasmic brown color. TLR5 immunopositivity was detected on the nuclear membranes and TLR7 as granular cytoplasmic brown immunopositivity (Fig. 1). We scored four cores from each tumor, choosing the core with the highest score for the statistical analysis. The results from both assessors were compared, and any intra-observer disagreements were reevaluated, with the final result achieved through discussion and consensus.

\section{Statistical analyses}

We used the Pearson's chi-square test to evaluate associations and Spearman's correlation test for correlations. Variables were dichotomized for statistical analysis as described below. The cutoff value for CRP was determined by the maximum value for Youden's index. We defined DSS as the time from surgery until death from CRC or until the end of the follow-up period. Survival curves were constructed using the Kaplan-Meier method and compared groups using the log-rank test. The 95\% confidence intervals (CIs) were calculated for the survival rates.

We used the Cox proportional hazards model to calculate the hazard ratios (HRs) for the uniand multivariate survival analyses. For the multivariate analysis, age, gender, tumor stage, and tumor location were used as independent covariates. Tumor stage was processed as a categorical covariate. A two-sided test with $p<0.05$ was considered significant for all analyses. All statistical analyses 
were performed using SPSS version 25.0 (IBM SPSS Statistics, version 25.0 for Mac; SPSS, Inc., Chicago, IL, USA, an IBM Company).

\section{Results}

\section{Immunostaining of TLRs}

From the 549 TMA samples, TLR2 immunoexpression was scored successfully in 541 (98.5\%), TLR4 in 537 (97.8\%), TLR5 in 539 (98.2\%), and TLR7 in $539(98.2 \%)$ cases. In a few cases, a TLR score was not possible to determine given missing representative cancer tissue or a technical failure. The score distributions for TLRs 2, 4, 5, and 7 are detailed in Supplementary Table 1.

For the statistical analysis, we dichotomized patients as follows: TLR2 low (scores 0-1) and high (scores 2-3; Figs. 1a-d), TLR4 low (scores 0-1) and high (scores 2-3; Figs. 1e-h), TLR5 low (scores 0-2) and high (score 3; Figs. 1i-m), and TLR7 negative (score 0) and positive (scores 1-3; Figs. 1n-r).

\section{Associations between TLRs, CRP, and clinicopathological parameters}

A low TLR5 associated with a higher tumor stage $(p<0.001$; Table 2$)$, higher pT classification $(p<$ 0.001; Table 2), and lymph node positivity ( $p=0.004$; Table 2). Positive TLR7 associated with leftsided disease ( $p=0.003$; Table 2$)$, a lower tumor stage $(p<0.001$; Table 2$)$, lower pT classification $(p=0.043$; Table 2), lower WHO grade $(p=0.035$; Table 2$)$, and with pN0 disease $(p<0.001$; Table 2). TLR2 and TLR4 did not associate with any clinicopathological parameters (Table 1). CRP was lower in stage I-III patients $(p<0.001$, Supplementary Table 2$)$ and patients with pT1-2 disease $(p$ $<0.001$, Supplementary Table 2). We observed no association between TLRs, age, gender, and CRP (Tables 1 and 2).

A high TLR2 immunoexpression associated with a high expression of all other TLRs $(p<$ 0.001 for all; data not shown) and comparisons also revealed weak or moderate positive correlations 
$(p<0.001$ for all; Table 3$)$. A high TLR4 expression associated with a high TLR5 expression $(p=$ 0.004; data not shown) and a positive TLR7 expression ( $p<0.001$; data not shown), also here weak positive correlations revealed ( $p<0.001$ for both, Table 3$)$. TLR5 did not associate with TLR7 ( $p=$ 0.266; data not shown), although we noted a weak positive correlation ( $p=0.006$, Table 3 ). No correlations emerged between TLRs and CRP (Table 3).

\section{Survival analysis}

The 5-year DSS reached 55.9\% among patients with a low TLR2 expression (95\% CI 44.9-68.9), compared to $71.2 \%(95 \%$ CI $66.9-75.5 ; p=0.005$; Fig. 2a) among those with a high TLR2 expression. TLR4 immunoexpression exhibited no prognostic value (HR 0.92; 95\% CI 0.67-1.22; $p$ $=0.504$; Fig 2b). Among patients with a low TLR5 expression, 5-year DSS reached $63.5 \%(95 \%$ CI 57.8-69.2) and 77.5\% (95\% CI 71.6-83.4; $p=0.002$, Fig. 2c) among patients with a high TLR5 expression. Among negative TLR7 immunoexpression patients, 5-year DSS fell to $51.1 \%$ (95\% CI 38.0-64.2), compared to 71.1\% (95\% CI 67.4-76.0; $p<0.001$; Fig. 2d) among those with a positive TLR7 expression. Among patients with a low CRP, 5-year DSS was 74.1\% (95\% CI 68.8-79.4) compared to $64.3 \%$ among high CRP patients (95\% CI 54.6-72.2; $p=0.017$; Fig. 2e).

In the subgroup analysis, among patients with stage III disease (HR 0.40; 95\% CI 0.24-0.65; $p<0.001$; Supplementary Table 3 ), a lower WHO grade (HR 0.73; 95\% CI 0.35-0.80; $p=0.003$; Supplementary Table 3), a younger age (HR 0.47; 95\% CI 0.26-0.83; $p=0.009$; Supplementary Table 3), female gender (HR 0.40; 95\% CI 0.25-0.67; $p<0.001$; Supplementary Table 3), a higher pT stage (HR 0.61; 95\% CI 0.40-0.94; $p=0.024$; Supplementary Table 3), and a low CRP (HR 0.53; 95\% CI 0.35-0.80; $p=0.002$; Fig. 3a and Supplementary Table 3), a high TLR2 immunoexpression served as positive prognostic factors.

Among patients with a high CRP (HR 2.04; 95\% CI 1.04-4.00; $p=0.038$; Fig. 3d and Supplementary Table 3), those with a high TLR4 immuoexpression exhibited a worse prognosis. 
Among patients with a lower WHO grade (HR 0.59; 95\% CI $0.41-0.83 ; p=0.003$; Supplementary Table 4), female gender (HR 0.52; 95\% CI 0.34-0.86; $p=0.009$; Supplementary Table 4), a higher pT stage (HR 0.67; 95\% CI 0.48-0.94; $p=0.020$; Supplementary Table 4), location in the rectum (HR 0.54; 95\% CI 0.34-0.86; $p=0.009$; Supplementary Table 4), and a low CRP (HR 0.059; 95\% CI 0.37-0.92; $p=0.021$; Fig. 3e and Supplementary Table 4), those with a high TLR5 immunoexpression experienced a better prognosis.

Patients with a positive TLR7 immunoexpression exhibited a better prognosis among subgroups of older patients (HR 0.47; 95\% CI 0.29-0.75; $p=0.002$; Supplementary Table 4), female gender (HR 0.38; 95\% CI 0.22-0.64; $p<0.001$; Supplementary Table 4), a lower WHO grade (HR 0.53; 95\% CI 0.33-0.86; $p=0.010$; Supplementary Table 4), a higher pT stage (HR 0.59; 95\% CI $0.39-0.89 ; p=0.012$; Supplementary Table 4), location in the right colon (HR $0.32 ; 95 \%$ CI $0.17-$ 0.60; $p<0.001$; Supplementary Table 4), and among patients with a low CRP (HR 0.53; 95\% CI 0.28-1.00; $p=0.049$; Fig. 3g and Supplementary Table 3).

In the multivariate Cox regression analysis including age, gender, tumor location, and tumor stage as covariates, none of the biomarkers under investigation served as significant independent factors of DSS, while age and tumor stage significantly associated with survival (data not shown).

\section{Discussion}

The prognostic role and possible relationship between the tissue immunoexpression of TLRs (local inflammatory response) and plasma CRP (SIR) among CRC patients have not previously been studied. Here, we observed a favorable prognosis among patients with a high TLR2 expression, a high TLR5 expression, a positive TLR7 expression, and a low CRP. We did not detect any association or correlation between the different TLRs and CRP. However, among patients with a low CRP, those with a high TLR2, TLR5, and TLR7 tumor expression exhibited a better prognosis and among high CRP patients, those with a low TLR4 immunoexpression exhibited a better prognosis. Unfortunately, 
none of the biomarkers examined remained independent prognostic factors in the multivariate analysis.

The biological mechanisms behind and interaction between systemic and local inflammation and carcinogenesis are complex and remain poorly understood. In epidemiological studies, an elevated level of circulating CRP serves as a marker of cancer as well as indicating a predisposition to the development of future malignancies [25]. Yet, some genetic epidemiological studies do not agree with this theory [25]. CRC patients with an increased CRP $(14,15,26)$, high mGPS [18,26], an elevated neutrophil-to-lymphocyte ratio (NLR) [27], elevated lymphocyte-to-monocyte ratio (LMR) [28), elevated platelet-to-lymphocyte ratio (PLR) [29], high IL-6 [30], and elevated tissue inhibitor of metalloproteinase-1 (TIMP-1) [31], all markers of SIR, associate with a worse prognosis.

Among many malignancies including $\mathrm{CRC}$, local and systemic inflammation have been studied separately, with very few studies assessing the association between local inflammation and SIR. Paarnio et al. studied both the serum and tumoral expression of TLR2 and TLR4 finding no association with each other, although patients with detectable serum TLR2 levels exhibited higher TLR2 immunoexpression in the normal colorectal mucosa [13]. They found that patients with undetectable TLR2 serum levels had higher CRP values, indicative of some connection between local and systemic inflammation [13]. Neither serum nor tissue TLR2 nor TLR4 expressions carried a prognostic value in their survival analysis $[13,32]$. However, they did not investigate the association between the tissue expression of TLRs and SIR as we did here. In our study, among the high CRP subgroup, patients with a low tumor TLR4 immunoexpression exhibited a better prognosis. In addition, a high TLR2, a high TLR5 and a positive TLR7 expression linked with a better prognosis among patients with low CRP, further suggesting a connection between local and systemic inflammation.

TLRs play an important role in carcinogenesis, although that role varies since the same TLR can act differently in differing malignancies [10]. Very few studies exist describing the role of TLRs 
in CRC. We previously demonstrated that patients with lymph node-positive disease with a strong tissue expression of TLR2 exhibited a better DSS [11]. The current study supports this finding, since CRC cases with a high TLR2 expression exhibited a better prognosis across the entire cohort. As mentioned above, Paarnio et al. observed no significant association between TLR2 and TLR4 tissue expressions and prognosis [32]. In our previous work, a strong TLR4 expression indicated a worse prognosis among Dukes B CRC patients [11], although such patients constituted a quite small proportion of cases in the present study, possibly explaining why TLR4 expression did not associate with prognosis. This finding agrees with previous work by Paarnio et al. [32].

In addition, we have demonstrated that a high TLR 5 expression serves as a positive prognostic factor in a cohort of 825 CRC patients [12], with the current study supporting this finding, since patients with a high TLR5 expression exhibited a better prognosis, also in the low CRP subgroup as well. Previously, we found no association between TLR7 immunoexpression and survival [12], despite patients with a positive TLR7 immunoexpression in the current study experiencing a better prognosis. Perhaps this discrepancy results from a difference in the study population, since in current study we included only patients for whom CRP information was available. In addition, the antibodies we used for immunostaining differed between the previous and the current study. A similar finding for the TLR7 association with a better prognosis was noted among stage III gastric cancer patients [33] and in patients with nasopharyngeal [34] and oropharyngeal tumors [35].

Guidelines for CRC treatment rely on TNM (tumor, node, and metastasis) staging, which takes into account only the tumor-associated factors and does not include the host's immune responses [36,37]. Patients with the same TNM stage may have different prognoses. For instance, in some patients, we see a relapse soon after curative intent treatment, while others may have stable disease for years or even show regression of their lymph node-positive or metastasized disease without additional treatment [38,39]. The diagnosis and treatment of stage II and III tumors remains under debate, since $17 \%$ of stage II and $36 \%$ of stage III patients relapse within 5 years $[2,38]$. This 
variation in outcomes among patients with the same stage disease shows that malignancies are complex and depend not only on tumor-related factors, but also on the tumor's interaction with its microenvironment and the host's immune response to the tumor. When the host's immunosurveillance mechanisms over-compete, the tumor begins to progress and metastasize [39]. TLRs as the first-line immune activators certainly play a role in immunosurveillance. In our present study, TLRs and CRP associate with prognosis, TLR2 specifically associating with prognosis among patients with stage III disease. Further studies are necessary in order to clarify the exact role of and interaction between local TLR immunoexpressions and SIR manifested as an elevated CRP in CRC, especially among patients whose disease recurs.

This large and well-characterized patient cohort with a long follow-up time represents a strength to our study. Missing reliable data about neoadjuvant or adjuvant therapy, however, can be seen as a limitation. Using TMA for immunohistochemistry is sometimes criticized, although this technique also carries several advantages, enabling the study of large numbers of immunostained samples. Punching tumor cores from several locations within the tumor sample overcomes the risk of misinterpretation because of the focal expression of antigens.

\section{Conclusions}

To our knowledge, this is the first study to evaluate the association between tissue TLR expression and CRP. A high tissue TLR2, high TLR5, positive TLR7, and low plasma CRP levels all associated with a better prognosis. However, no association or correlation was observed between different TLRs and CRP. Further research is still needed to understand the role of TLRs in CRC and the interaction between systemic inflammation and the local activation of TLRs to better understand tumor development.

\section{Notes}

\section{Acknowledgments}


We thank Pia Saarinen for technical assistance with laboratory methods, and Vanessa Fuller for exceptional Englishlanguage revision. We thank the funders mentioned below for their financial support of this research.

\section{Statement of Ethics}

The Surgical Ethics Committee of Helsinki University Hospital (Dnro HUS 226/E6/06, extension TMK02 §66 17.4.2013) approved the study protocol. The National Supervisory Authority of Health and Welfare (Valvira Dnro 10041/06.01.03.01/2012) permitted the use of archival tissue and blood samples without requiring individual consent. The study complied with the guidelines for human studies and was conducted ethically in accordance with the World Medical Association Declaration of Helsinki.

\section{Funding}

This study was financially supported by the Competitive State Research Financing of the Expert Responsibility Area of Helsinki University Hospital (CH), the Finnish Cancer Foundation (CH), Finska Läkaresällskapet (IB-L, CB), the Sigrid Jusélius Foundation (CH) and the Faculty of Medicine, University of Helsinki, Helsinki, Finland (IBL, CB), the Kurt och Doris Palander Foundation (IB-L), the K. Albin Johansson Foundation (CB, IB-L). The funders played no role in the study design, analysis, the interpretation of data, publishing decision, or in writing the manuscript.

\section{Conflicts of Interest Statement}

The authors have no conflicts of interest to declare.

\section{Author Contributions}

IB-L, CB, CH, JH, UG, RP, and KS participated in conceptualizing and designing the study. CH collected the blood and tissue samples. IB-L and TK collected and processed the data. JH contributed to the specimen preparation. IB-L and JH scored the samples. Serum samples were analyzed by US. IB-L analyzed the results, drafted the manuscript, and prepared the original figures and tables. All authors agreed to the content of the manuscript, and provided their final approval of the current version of the submitted manuscript. $\mathrm{CB}$ and $\mathrm{CH}$ equally participated in supervision.

\section{Figure legends}

Fig. 1. Images of the TLR2, TLR4, TLR5, and TLR7 immunohistochemistry stainings representing colorectal cancer tumors with negative $(\mathbf{a}, \mathbf{e}, \mathbf{i}, \mathbf{m})$, mild $(\mathbf{b}, \mathbf{f}, \mathbf{k}, \mathbf{o})$, moderate $(\mathbf{c}, \mathbf{g}, \mathbf{l}, \mathbf{p})$, and strong $(\mathbf{d}, \mathbf{h}, \mathbf{m}, \mathbf{r})$ staining. Original magnification: 20x. 
Fig. 2. Colorectal cancer patients' disease-specific survival analysis for different TLRs and CRP using the Kaplan-Meier method; (a) TLR2, (b) TLR4, (c) TLR5 and TLR7 (d), and CRP (e). $p$ value for the log-rank test.

Fig. 3. Colorectal cancer patients' disease-specific survival analysis by subgroup using the KaplanMeier method. A high versus low TLR2 among patients with (a) a low CRP and (b) a high CRP. A high versus low TLR4 among patients with (c) a low CRP and (d) a high CRP. A high versus low TLR5 among patients with (e) a low CRP and (f) a high CRP. A positive versus negative TLR7 among patients with (g) a low CRP and (h) a high CRP. $p$ value for the log-rank test.

\section{References}

1. Bray F, Ferlay J, Soerjomataram I, Siegel RL, Torre LA, Jemal A. Global cancer statistics 2018: GLOBOCAN estimates of incidence and mortality worldwide for 36 cancers in 185 countries. CA Cancer J Clin. 2018 Nov;68(6):394-424.

2. Böckelman C, Engelmann BE, Kaprio T, Hansen TF, Glimelius B. Risk of recurrence in patients with colon cancer stage II and III: A systematic review and meta-analysis of recent literature. Acta Oncologica. 2014 Sep 29;54(1):5-16.

3. Canavan C, Abrams KR, Mayberry J. Meta-analysis: colorectal and small bowel cancer risk in patients with Crohn's disease. Aliment Pharmacol Ther. John Wiley \& Sons, Ltd; 2006 Apr 15;23(8):1097-104.

4. Sun G, Dong X, Tang X, Qu H, Zhang H, Zhao E. The prognostic value of immunoscore in patients with colorectal cancer: A systematic review and meta-analysis. Cancer Med. John Wiley \& Sons, Ltd; 2019 Jan;8(1):182-9.

5. El-Zayat SR, Sibaii H, Mannaa FA. Toll-like receptors activation, signaling, and targeting: an overview. Bulletin of the National Research Centre; 2019 Dec 11;:1-12.

6. Sato Y, Goto Y, Narita N, Hoon DSB. Cancer Cells Expressing Toll-like Receptors and the Tumor Microenvironment. Cancer Microenviron. Springer Netherlands; 2009 Sep;2 Suppl 1(S1):205-14.

7. Chen K, Huang J, Gong W, Iribarren P, Dunlop NM, Wang JM. Toll-like receptors in inflammation, infection and cancer. Int Immunopharmacol. 2007 Oct;7(10):1271-85.

8. Iwasaki A, Medzhitov R. Toll-like receptor control of the adaptive immune responses. Nat Immunol. 2004 Oct 1;5(10):987-95. 
9. Pradere J-P, Dapito DH, Schwabe RF. The Yin and Yang of Toll-like receptors in cancer. Oncogene. 2013 Aug 12;33(27):3485-95.

10. Dajon M, Iribarren K, Cremer I. Toll-like receptor stimulation in cancer: A pro- and antitumor double-edged sword. Immunobiology. Elsevier GmbH; 2017 Jan 1;222(1):89-100.

11. Beilmann-Lehtonen I, Böckelman C, Mustonen H, Koskensalo S, Hagström J, Haglund C. The prognostic role of tissue TLR2 and TLR4 in colorectal cancer. Virchows Arch. Springer Berlin Heidelberg; 2020 Nov;477(5):705-15.

12. Beilmann-Lehtonen I, Hagström J, Mustonen H, Koskensalo S, Haglund C, Böckelman C. High tissue TLR5 expression predicts better survival in colorectal cancer patients. Oncology; 2021 Jun 17:1-12. doi: 10.1159/000516543. Epub ahead of print.

13. Paarnio K, Tuomisto A, Väyrynen SA, Väyrynen JP, Klintrup K, Ohtonen P, et al. Serum TLR2 and TLR4 levels in colorectal cancer and their association with systemic inflammatory markers, tumor characteristics, and disease outcome. APMIS. John Wiley \& Sons, Ltd; 2019 Jul 19;127(8):561-9.

14. Kersten C, Louhimo J, Ålgars A, Lahdesmaki A, Cvancerova M, Stenstedt K, et al. Increased C-reactive protein implies a poorer stage-specific prognosis in colon cancer. Acta Oncologica. 2013 Oct 9;52(8):1691-8.

15. Køstner AH, Kersten C, Löwenmark T, Ydsten KA, Peltonen R, Isoniemi H, et al. The prognostic role of systemic inflammation in patients undergoing resection of colorectal liver metastases: C-reactive protein (CRP) is a strong negative prognostic biomarker. J Surg Oncol. 2016 Oct 3;114(7):895-9.

16. Nozoe T, Mori E, Takahashi I, Ezaki T. Preoperative elevation of serum C-reactive protein as an independent prognostic indicator of colorectal carcinoma. Surg Today. Springer Japan; 2008 Jul 9;38(7):597-602.

17. Kim WR, Han YD, Min BS. C-Reactive Protein Level Predicts Survival Outcomes in Rectal Cancer Patients Undergoing Total Mesorectal Excision After Preoperative Chemoradiation Therapy. Ann Surg Oncol. Springer International Publishing; 2018 Oct 4;25(13):3898-905.

18. Lu X, Guo W, Xu W, Zhang X, Shi Z, Zheng L, et al. Prognostic value of the Glasgow prognostic score in colorectal cancer: a meta-analysis of 9,839 patients. Cancer Manag Res. Dove Press; 2019; Volume 11:229-49.

19. Canna K, McArdle PA, McMillan DC, McNicol A-M, Smith GW, McKee RF, et al. The relationship between tumour T-lymphocyte infiltration, the systemic inflammatory response and survival in patients undergoing curative resection for colorectal cancer. British Journal of Cancer. Nature Publishing Group; 2005 Feb 8;92(4):651-4.

20. Gunnarsson U, Strigård K, Edin S, Gkekas I, Mustonen H, Kaprio T, et al. Association between local immune cell infiltration, mismatch repair status and systemic inflammatory response in colorectal cancer. J Transl Med. BioMed Central; 2020 Apr 21;18(1):178-8.

21. Roxburgh CSD, Salmond JM, Horgan PG, Oien KA, McMillan DC. The Relationship Between the Local and Systemic Inflammatory Responses and Survival in Patients 
Undergoing Curative Surgery for Colon and Rectal Cancers. J Gastrointest Surg. SpringerVerlag; 2009 Sep 19;13(11):2011-9.

22. AJCC Cancer Staging Manual. 6th edition Springer; New York (NY): 2002

23. Salmiheimo A, Mustonen H, Stenman U-H, Puolakkainen P, Kemppainen E, Seppänen H, et al. Systemic Inflammatory Response and Elevated Tumour Markers Predict Worse Survival in Resectable Pancreatic Ductal Adenocarcinoma. Coleman WB, editor. PLoS ONE. Public Library of Science; 2016;11(9):e0163064.

24. Kallioniemi OP, Wagner U, Kononen J, Sauter G. Tissue microarray technology for highthroughput molecular profiling of cancer. Hum Mol Genet. 2001 Apr;10(7):657-62.

25. Allin KH, Nordestgaard BG. Elevated C-reactive protein in the diagnosis, prognosis, and cause of cancer. Crit Rev Clin Lab Sci. 2011 Jul;48(4):155-70.

26. Woo HD, Kim K, Kim J. Association between preoperative C-reactive protein level and colorectal cancer survival: a meta-analysis. Cancer Causes Control. Springer International Publishing; 2015 Sep 16;26(11):1661-70.

27. Li H, Zhao Y, Zheng F. Prognostic significance of elevated preoperative neutrophil-tolymphocyte ratio for patients with colorectal cancer undergoing curative surgery: A metaanalysis. Medicine (Baltimore). 2019 Jan;98(3):e14126.

28. Tan D, Fu Y, Tong W, Li F. Prognostic significance of lymphocyte to monocyte ratio in colorectal cancer: A meta-analysis. Int J Surg. 2018 Jul;55(1):128-38.

29. Tan D, Fu Y, Su Q, Wang H. Prognostic role of platelet-lymphocyte ratio in colorectal cancer: A systematic review and meta-analysis. Medicine (Baltimore). 2016 Jun;95(24):e3837.

30. Xu J, Ye Y, Zhang H, Szmitkowski M, Mäkinen MJ, Li P, et al. Diagnostic and Prognostic Value of Serum Interleukin-6 in Colorectal Cancer. Medicine (Baltimore). 2016 Jan;95(2):e2502.

31. Lee J-H, Choi J-W, Kim Y-S. Plasma or serum TIMP-1 is a predictor of survival outcomes in colorectal cancer: a meta-analysis. J Gastrointestin Liver Dis. 2011 Sep;20(3):287-91.

32. Paarnio K, Väyrynen S, Klintrup K, Ohtonen P, Mäkinen MJ, Mäkelä J, et al. Divergent expression of bacterial wall sensing Toll-like receptors 2 and 4 in colorectal cancer. WJG. 2017 Jul 14;23(26):4831-8.

33. Kasurinen A, Hagström J, Laitinen A, Kokkola A, Böckelman C, Haglund C. Evaluation of toll-like receptors as prognostic biomarkers in gastric cancer: high tissue TLR5 predicts a better outcome. Sci Rep. Nature Publishing Group; 2019 Aug 29;9(1):12553-10.

34. Ruuskanen M, Leivo I, Minn H, Vahlberg T, Haglund C, Hagström J, et al. Expression of toll-like receptors in non-endemic nasopharyngeal carcinoma. BMC Cancer. 4 ed. 2019 Jun 25;19(1):335-12.

35. Jouhi L, Mohamed H, Mäkitie A, Remes SM, Haglund C, Atula T, et al. Toll-like receptor 5 and 7 expression may impact prognosis of HPV-positive oropharyngeal squamous cell 
carcinoma patients. Cancer Immunol Immunother. 4 ed. Springer Berlin Heidelberg; 2017 Dec;66(12):1619-29.

36. Nagtegaal ID, Quirke P, Schmoll H-J. Has the new TNM classification for colorectal cancer improved care? Nat Rev Clin Oncol. Nature Publishing Group; 2011 Oct 18;9(2):119-23.

37. Weiser MR. AJCC 8th Edition: Colorectal Cancer. Ann Surg Oncol. Springer International Publishing; 2018 Jun;25(6):1454-5.

38. Tsikitis VL, Larson DW, Huebner M, Lohse CM, Thompson PA. Predictors of recurrence free survival for patients with stage II and III colon cancer. BMC Cancer. 2014 May $16 ; 14(1): 10-7$.

39. Mlecnik B, Bindea G, Pagès F, Galon J. Tumor immunosurveillance in human cancers. Cancer Metastasis Rev. 6 ed. Springer US; 2011 Jan 21;30(1):5-12. 


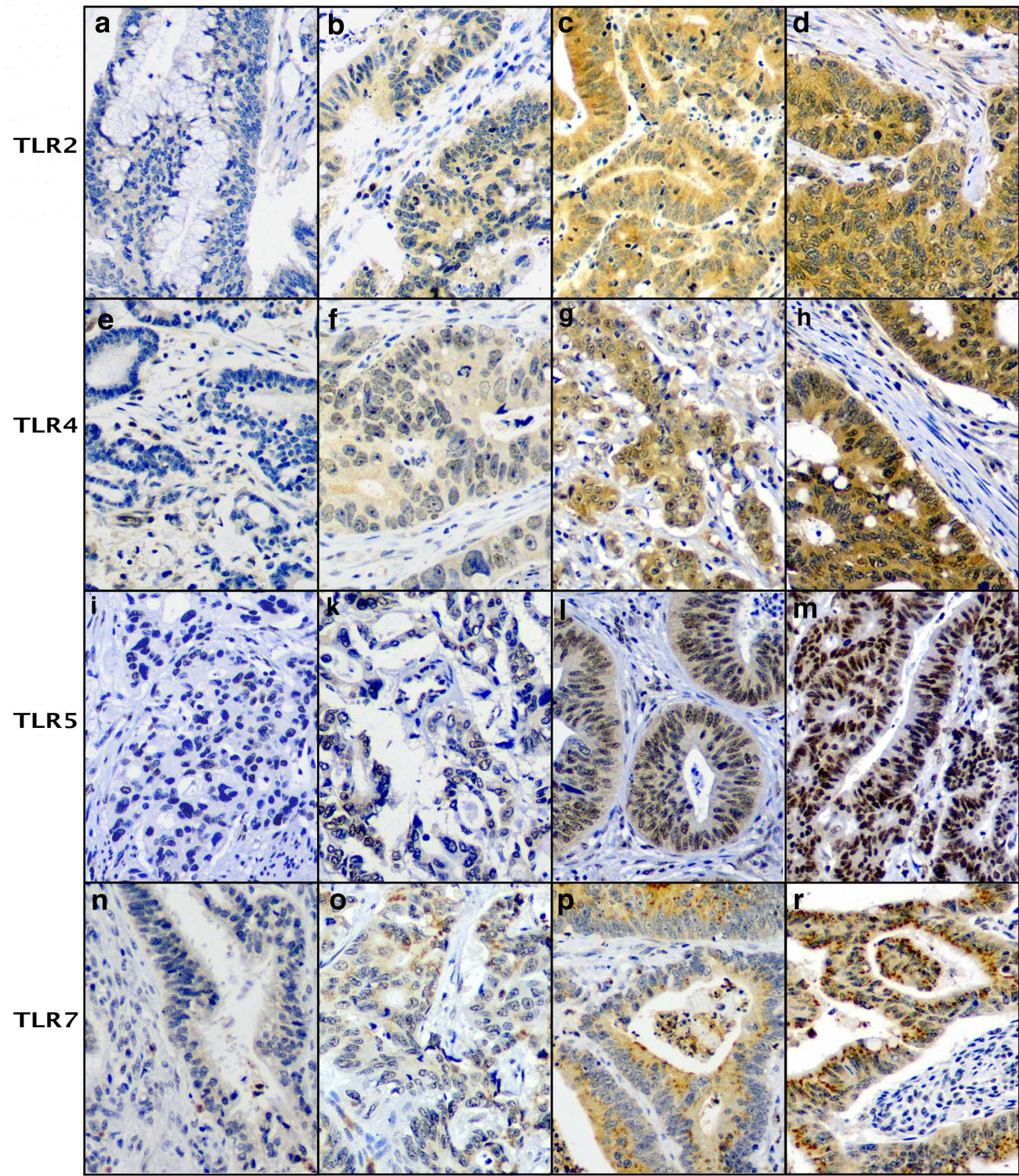


a

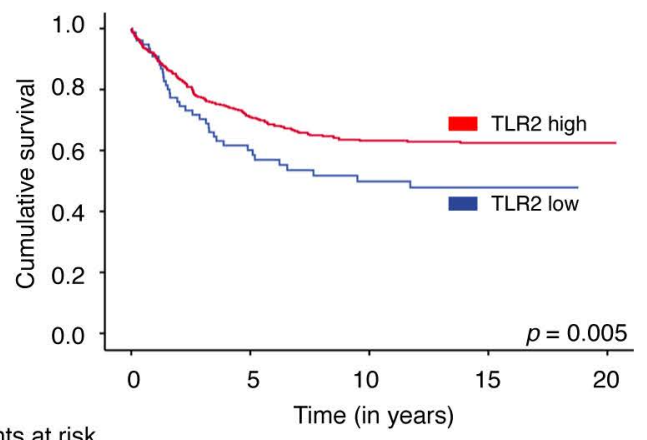

Patients at risk

TLR2 low:

TLR2 high: b

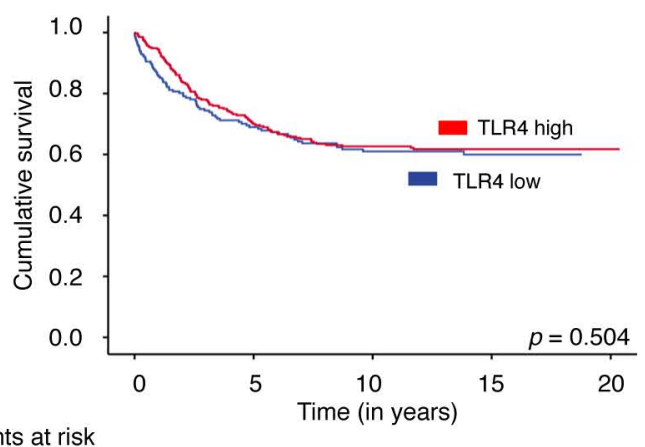

Patients at risk

TLR4 low:

TLR4 high: $\begin{array}{lllll}212 & 123 & 86 & 36 & 0\end{array}$
C

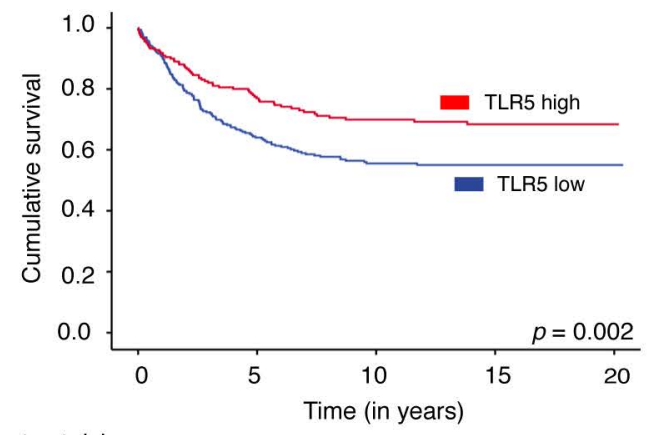

Patients at risk

$\begin{array}{llllll}\text { TLR5 low: } & 314 & 161 & 116 & 66 & 2 \\ \text { TLR5 high: } & 223 & 144 & 103 & 59 & 1\end{array}$

d

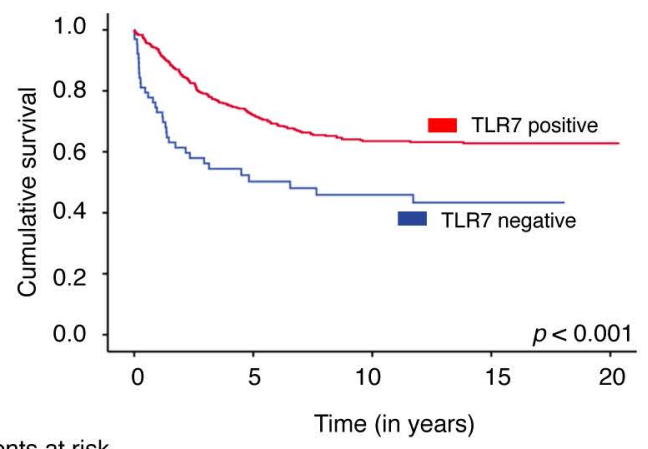

Patients at risk

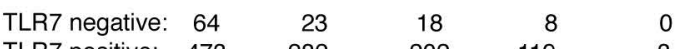

e

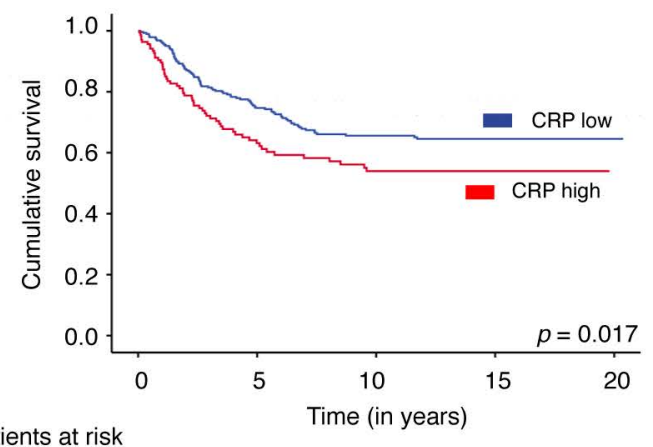

Patients at risk

$\begin{array}{lrrrrr}\text { CRP low: } & 286 & 180 & 129 & 70 & 3 \\ \text { CRP high: } & 139 & 64 & 48 & 35 & 0\end{array}$




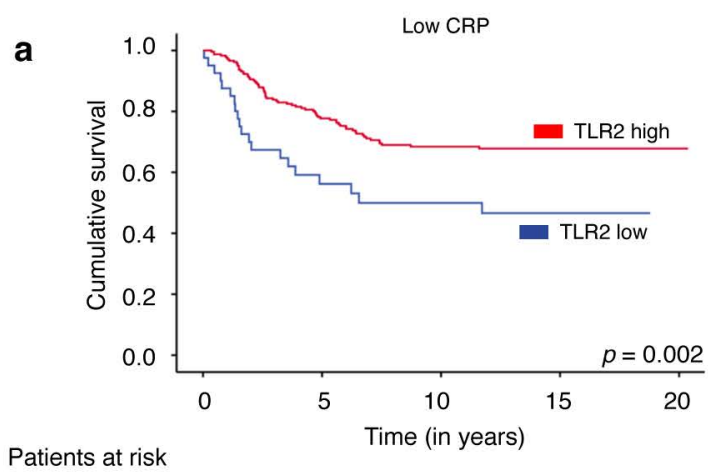

Patients at risk

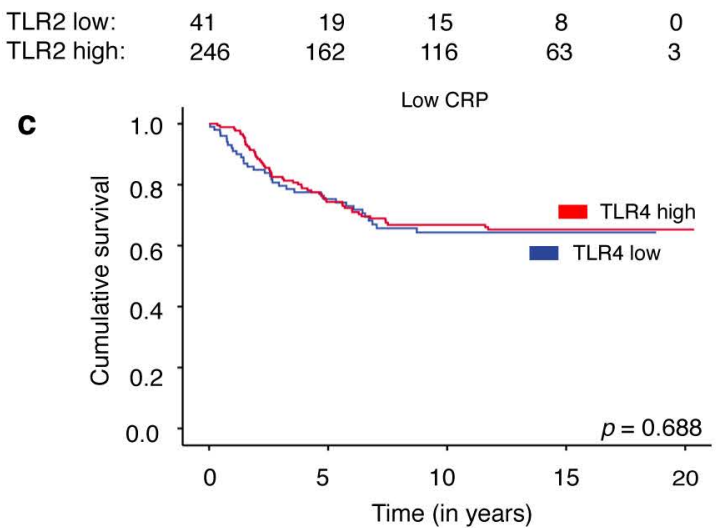

Patients at risk

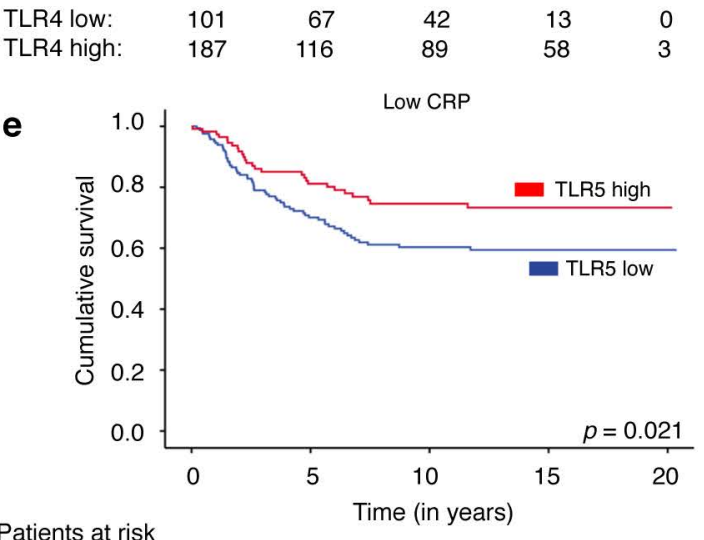

Patients at risk

TLR5 low: $\quad 167$

TLR5 high: $\quad 120$

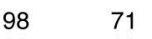

83

60

37

34

Low CRP

9

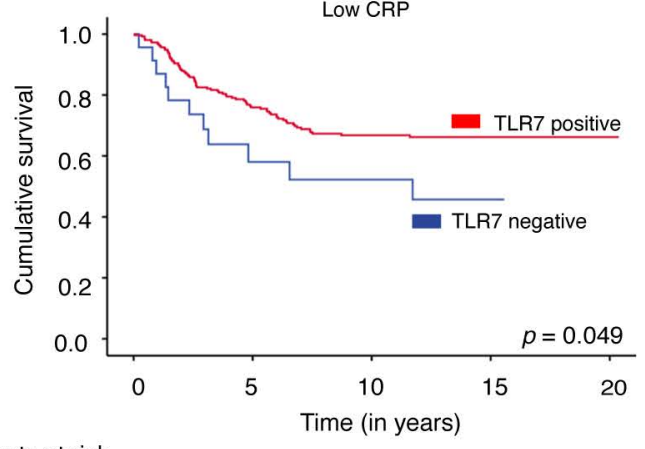

Patients at risk

TLR7 negative: 23

TLR7 positive: 263
10

170 b

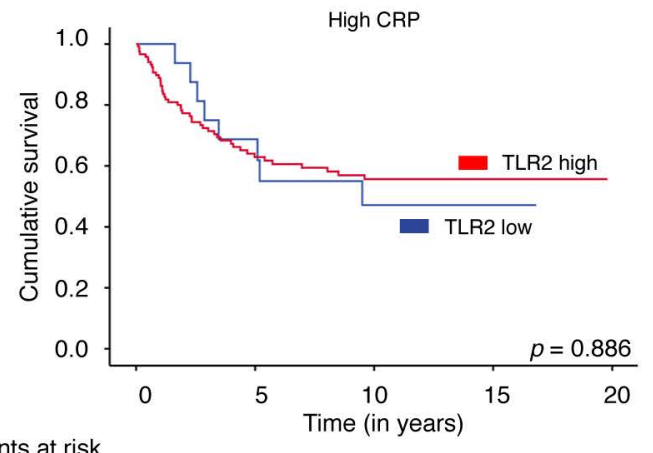

Patients at risk

$\begin{array}{lrrrcc}\text { TLR2 low: } & 16 & 10 & 6 & 5 & 0 \\ \text { TLR2 high: } & 125 & 56 & 43 & 30 & 0\end{array}$

d

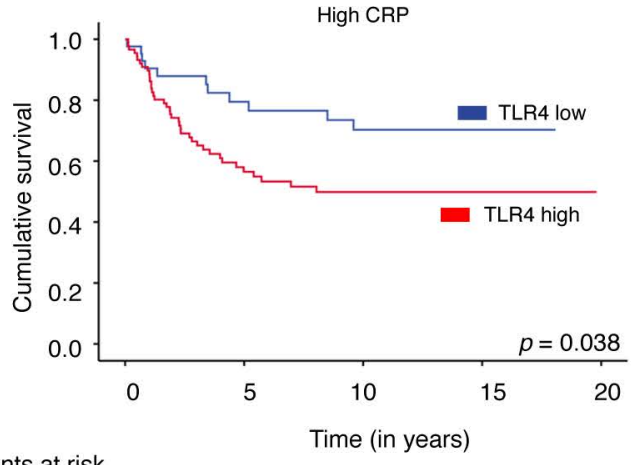

Patients at risk

$\begin{array}{lllll}44 & 27 & 22 & 14 & 0\end{array}$

$\begin{array}{llllll}\text { TLR4 high: } & 93 & 37 & 27 & 21 & 0\end{array}$

f

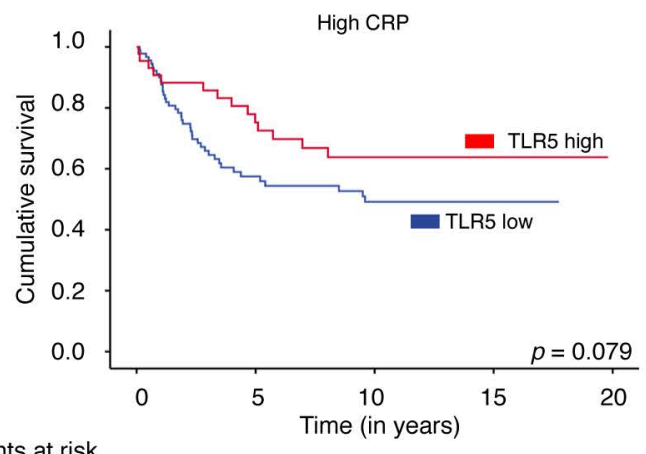

Patients at risk

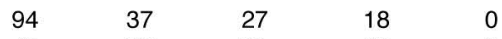

$\begin{array}{llllll}\text { TLR5 high: } & 46 & 28 & 21 & 21 & 0\end{array}$

h

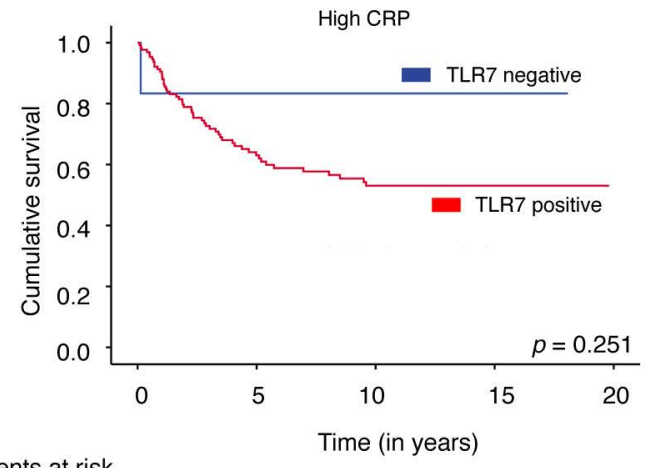

Patients at risk

TLR7 negative:

TLR7 positive: 132

$\begin{array}{ccrc}5 & 5 & 5 & 0 \\ 61 & 44 & 31 & 0\end{array}$


Table 1. Association between TLR2 and TLR4 immunointensity and clinicopathological variables among 549 colorectal cancer patients

\begin{tabular}{|c|c|c|c|c|c|c|c|}
\hline \multirow{2}{*}{$\begin{array}{l}\text { Clincopathological } \\
\text { variable }\end{array}$} & \multirow[b]{2}{*}{ n (\%) } & \multicolumn{3}{|c|}{ TLR2 } & \multicolumn{3}{|c|}{ TLR4 } \\
\hline & & Low $(\%)$ & High (\%) & $p$ value $^{1}$ & Low $(\%)$ & High (\%) & $p$ value $^{1}$ \\
\hline \multicolumn{8}{|l|}{ Age } \\
\hline$<65$ & $220(40.1)$ & $28(13.0)$ & $188(87.9)$ & 0.847 & $84(39.4)$ & $129(60.6)$ & 0.412 \\
\hline$\geq 65$ & 329 (59.9) & $44(13.5)$ & $281(86.5)$ & & $116(35.8)$ & 208 (64.2) & \\
\hline
\end{tabular}

Gender

$\begin{array}{llllllll}\text { Male } & 289(52.6) & 38(13.4) & 245(86.6) & 0.932 & 98(34.8) & 184(65.2) & 0.212 \\ \text { Female } & 260(47.4) & 34(13.2) & 224(86.8) & & 102(40.0) & 153(60.0) & \end{array}$

Location

$\begin{array}{llllllll}\text { Right colon } & 155(28.2) & 14(9.3) & 137(90.7) & 0.073 & 53(35.1) & 98(64.9) & 0.783 \\ \text { Left colon } & 126(23.0) & 14(11.1) & 112(88.9) & & 46(37.1) & 78(62.9) & \\ \text { Rectum } & 268(48.8) & 44(16.7) & 220(83.3) & & 101(38.5) & 161(61.5) & \end{array}$

Tumor stage

$\begin{array}{llllllll}\text { I } & 108(19.7) & 14(13.2) & 92(86.8) & 0.481 & 37(34.6) & 70(65.4) & 0.840 \\ \text { II } & 153(27.9) & 15(9.9) & 137(90.1) & & 58(38.4) & 93(61.6) & \\ \text { III } & 201(36.7) & 28(14.1) & 170(85.9) & & 75(38.7) & 119(61.3) & \\ \text { IV } & 86(15.7) & 14(16.7) & 70(83.3) & & 29(34.5) & 55(65.5)\end{array}$

Tumor classification (pT)

$\begin{array}{llllllll}\text { pT1-pT2 } & 134(24.8) & 16(12.1) & 116(7.9) & 0.768 & 49(36.8) & 84(63.2) & 0.520 \\ \text { pT3-pT4 } & 407(75.2) & 54(13.5) & 347(86.5) & & 147(37.1) & 249(62.9) & \end{array}$

Lymph node metastasis $(\mathrm{pN})$

$\begin{array}{llllllll}\text { pN0 } & 276(51.2) & 32(11.7) & 241(88.3) & 0.369 & 97(35.5) & 176(64.5) & 0.369 \\ \text { pN1-2 } & 263(48.8) & 38(14.7) & 220(85.3) & & 100(39.4) & 154(60.6) & \end{array}$

Tumor grade (WHO)

$\begin{array}{llllllll}1 & 43(8.7) & 3(7.1) & 39(92.9) & 0.522 & 14(32.6) & 29(67.4) & 0.515 \\ 2 & 389(78.9) & 52(13.4) & 335(86.6) & & 145(38.1) & 236(61.9) & \\ 3 & 29(5.9) & 4(13.8) & 25(86.29 & & 9(31.0) & 20(69.0) \\ 4 & 32(6 .) & 4(13.3) & 26(86.7) & & 8(26.7) & 22(73.3)\end{array}$

Systemic inflammatory response (CRP)

\begin{tabular}{llllllll}
$\leq 8.7$ & $287(67.4)$ & $41(14.5)$ & $242(85.5)$ & 0.453 & $97(34.3)$ & $186(65.7)$ & 0.739 \\
$>8.7$ & $139(32.6)$ & $16(11.7)$ & $121(88.3)$ & & $43(32.3)$ & $90(67.7)$ & \\
\hline
\end{tabular}

Abbreviations: CRP, C-reactive protein; TLR, toll-like receptor 
Table 2. Association between TLR5 and TLR7 immunointensity and clinicopathological variables among 549 colorectal cancer patients

\begin{tabular}{|c|c|c|c|c|c|c|}
\hline \multirow{2}{*}{$\begin{array}{l}\text { Clincopathological } \\
\text { variable }\end{array}$} & \multicolumn{3}{|c|}{ TLR5 } & \multicolumn{3}{|c|}{ TLR7 } \\
\hline & Low (\%) & High (\%) & $p$ value $^{1}$ & Negative (\%) & Positive (\%) & $p$ value $^{1}$ \\
\hline \multicolumn{7}{|l|}{ Age } \\
\hline$<65$ & $118(54.9)$ & $97(45.1)$ & 0.154 & $23(10.6)$ & $193(89.4)$ & 0.500 \\
\hline$\geq 65$ & $198(61.6)$ & $126(38.9)$ & & $41(12.79$ & $282(87.3)$ & \\
\hline \multicolumn{7}{|l|}{ Gender } \\
\hline Male & $164(58.4)$ & $117(41.6)$ & 0.930 & $34(12.1)$ & $248(87.9)$ & 0.499 \\
\hline Female & $152(58.9)$ & $106(41.1)$ & & $30(11.7)$ & $227(88.3)$ & \\
\hline \multicolumn{7}{|l|}{ Location } \\
\hline Right colon & $87(57.2)$ & $65(42.8)$ & 0.836 & $29(19.3)$ & $121(80.7)$ & 0.003 \\
\hline Left colon & $72(57.6)$ & $53(42.4)$ & & $13(10.3)$ & $113(89.7)$ & \\
\hline Rectum & $157(59.9)$ & $105(40.1)$ & & $22(8.4)$ & $241(91.6)$ & \\
\hline \multicolumn{7}{|l|}{ Tumor stage } \\
\hline I & $44(41.1)$ & $63(58.9)$ & $<0.001$ & $8(7.5)$ & $99(92.5)$ & $<0.001$ \\
\hline II & $89(58.9)$ & $62(41.1)$ & & $6(4.0)$ & $145(96.0)$ & \\
\hline III & $123(62.8)$ & $73(37.2)$ & & $33(16.8)$ & $163(83.2)$ & \\
\hline IV & $59(70.2)$ & $25(29.8)$ & & $16(19.0)$ & $68(81.0)$ & \\
\hline \multicolumn{7}{|c|}{ Tumor classification (pT) } \\
\hline pT1-pT2 & $54(40.6)$ & $79(59.4)$ & $<0.001$ & $9(6.8)$ & $124(93.2)$ & 0.043 \\
\hline pT3-pT4 & $260(65.3)$ & $138(34.7)$ & & $53(13.3)$ & $345(86.7)$ & \\
\hline \multicolumn{7}{|c|}{ Lymph node metastasis (pN) } \\
\hline pN0 & $146(53.5)$ & $127(46.5)$ & 0.004 & $14(5.1)$ & $259(94.9)$ & $<\mathbf{0 . 0 0 1}$ \\
\hline $\mathrm{pN} 1-2$ & $168(65.6)$ & $88(34.4)$ & & $47(18.4)$ & $209(81.6)$ & \\
\hline \multicolumn{7}{|l|}{ Tumor grade (WHO) } \\
\hline 1 & $24(55.8)$ & $19(44.2)$ & 0.966 & $4(9.3)$ & $39(90.7)$ & 0.035 \\
\hline 2 & $224(58.2)$ & $161(41.8)$ & & $37(9.6)$ & $348(90.4)$ & \\
\hline 3 & $18862.1)$ & $11(37.9)$ & & $6(21.4)$ & $22(78.6)$ & \\
\hline 4 & $17(58.6)$ & $12(41.4)$ & & $7(23.3)$ & $23(76.7)$ & \\
\hline \multicolumn{7}{|c|}{ Systemic inflammatory response (CRP) } \\
\hline$\leq 8.7$ & $165(58.3)$ & $118(41.7)$ & 0.135 & $23(8.2)$ & $258(91.8)$ & 0.435 \\
\hline$>8.7$ & $90(66.2)$ & $46(33.8)$ & & $8(5.9)$ & $128(94.1)$ & \\
\hline
\end{tabular}

Abbreviations: CRP, C-reactive protein; TLR, toll-like receptor

${ }^{1}$ Chi-square test 
Table 3. Correlations for TLR2, TLR4, TLR5, and TLR7 among each other and with CRP in 549 colorectal cancer patients

\begin{tabular}{|c|c|c|c|c|c|c|c|c|}
\hline & \multicolumn{2}{|c|}{ TLR2 } & \multicolumn{2}{|c|}{ TLR4 } & \multicolumn{2}{|c|}{ TLR5 } & \multicolumn{2}{|c|}{ TLR7 } \\
\hline & $\mathbf{r}_{\mathrm{s}}$ & $p$ value & $\mathbf{r}_{\mathrm{s}}$ & $p$ value & $\mathbf{r}_{\mathrm{s}}$ & $p$ value & $\mathbf{r}_{\mathrm{s}}$ & $p$ value \\
\hline TLR4 & 0.219 & $<0.001$ & & & & & & \\
\hline TLR5 & 0.157 & $<0.001$ & 0.317 & $<0.001$ & & & & \\
\hline TLR7 & 0.171 & $<0.001$ & 0.248 & $<0.001$ & 0.109 & 0.006 & & \\
\hline CRP & 0.069 & 0.161 & 0.033 & 0.505 & -0.039 & 0.421 & -0.012 & 0.813 \\
\hline
\end{tabular}

Abbreviations: CRP, C-reactive protein; TLR, toll-like receptor

$\mathrm{r}_{\mathrm{s}}=$ Spearmans's correlation coefficent 
Supplementary Table 1. Expression distribution for TLR2, TLR4, TLR5, and TLR7 among 549 colorectal cancer patients

\begin{tabular}{|c|c|c|c|c|}
\hline & Negative (\%) & Low (\%) & Moderate (\%) & Strong $(\%)$ \\
\hline TLR2 & $10(1.8)$ & $62(11.5)$ & $252(46.6)$ & $217(40.1)$ \\
\hline TLR4 & $48(8.9)$ & $152(28.3)$ & $246(45.8)$ & $91(16.9)$ \\
\hline TLR5 & $60(11.1)$ & $58(10.8)$ & $198(36.7)$ & $223(41.4)$ \\
\hline TLR7 & $64(11.9)$ & $175(32.5)$ & $248(46.0)$ & $52(9.6)$ \\
\hline
\end{tabular}

Abbreviations: TLR, toll-like receptor 
Supplementary Table 2. Association between CRP and clinicopathological parameters among 549 colorectal cancer patients

\begin{tabular}{|c|c|c|c|}
\hline \multirow{2}{*}{$\begin{array}{l}\text { Clincopathological } \\
\text { variable }\end{array}$} & \multicolumn{3}{|c|}{ CRP } \\
\hline & $\leq 8.7$ & $>8.7$ & $p$ value $^{1}$ \\
\hline \multicolumn{4}{|l|}{ Age } \\
\hline$<65$ & $119(64.7$ & $65(35.3)$ & 0.348 \\
\hline$\geq 65$ & $168(69.4)$ & $74(30.6)$ & \\
\hline
\end{tabular}

Gender

$\begin{array}{llll}\text { Male } & 140(69.3) & 62(30.7) & 0.469 \\ \text { Female } & 147(65.6 & 77(34.4) & \end{array}$

Location

$\begin{array}{llll}\text { Right colon } & 63(61.8) & 39(38.2) & 0.150 \\ \text { Left colon } & 51(63.0) & 30(37.0) & \\ \text { Rectum } & 173(71.2) & 70(28.8) & \end{array}$

Tumor stage

$\begin{array}{llll}\text { I } & 68(81.9) & 15(18.1) & <\mathbf{0 . 0 0 1} \\ \text { II } & 81(67.5) & 39(32.5) & \\ \text { III } & 107(68.6) & 49(31.4) & \\ \text { IV } & 31(46.3) & 36(53.7) & \end{array}$

Tumor classification (pT)

$\begin{array}{llll}\text { pT1-pT2 } & 86(81.9) & 19(18.1) & <\mathbf{0 . 0 0 1} \\ \text { pT3-pT4 } & 196(62.0) & 120(38.0) & \end{array}$

Lymph node metastasis $(\mathrm{pN})$

$\begin{array}{llll}\text { pN0 } & 155(71.1) & 63(28.9) & 0.062 \\ \text { pN1-2 } & 126(62.4) & 76(37.6) & \end{array}$

Tumor grade (WHO)

\begin{tabular}{llll}
1 & $24(70.6)$ & $10(29.4)$ & 0.316 \\
2 & $207(68.1)$ & $97(31.9)$ & \\
3 & $19(79.2)$ & $5(20.8)$ & \\
4 & $13(54.2)$ & $11(45.8)$ & \\
\hline
\end{tabular}

Abbreviations: CRP, C-reactive protein

${ }^{1}$ Chi-square test 
Supplementary Table 3. Survival analysis by subgroups, high TLR2 and TLR4 tissue expressions compared to low in 549 colorectal cancer patients

\begin{tabular}{|c|c|c|c|c|c|c|}
\hline & \multicolumn{3}{|c|}{ High TLR2 } & \multicolumn{3}{|c|}{ High TLR4 } \\
\hline & HR & $95 \%$ CI & $p$ value & HR & $95 \%$ CI & $p$ value \\
\hline \multicolumn{7}{|l|}{ Age } \\
\hline$<65$ & 0.47 & $0.26-0.83$ & 0.009 & 0.74 & $0.46-1.19$ & 0.209 \\
\hline$\geq 65$ & 0.69 & $0.43-1.11$ & 0.128 & 1.01 & $0.69-1.48$ & 0.949 \\
\hline
\end{tabular}

Gender

$\begin{array}{lllllll}\text { Male } & 0.85 & 0.50-1.45 & 0.541 & 1.22 & 0.80-1.87 & 0.357 \\ \text { Female } & 0.40 & 0.25-0.67 & \mathbf{< 0 . 0 0 1} & 0.66 & 0.43-1.00 & 0.050\end{array}$

Location

$\begin{array}{lllllll}\text { Right colon } & 0.55 & 0.25-1.24 & 0.149 & 0.56 & 0.31-1.02 & 0.058 \\ \text { Left colon } & 0.51 & 0.25-1.01 & 0.069 & 0.74 & 0.42-1.28 & 0.277 \\ \text { Rectum } & 0.63 & 0.38-1.04 & 0.070 & 1.35 & 0.87-2.10 & 0.185\end{array}$

Tumor stage

$\begin{array}{lllllll}\text { I } & 0.54 & 0.11-2.59 & 0.438 & 0.42 & 0.11-1.56 & 0.194 \\ \text { II } & 0.91 & 0.28-3.02 & 0.878 & 0.63 & 0.30-1.33 & 0.225 \\ \text { III } & 0.40 & 0.24-0.65 & <\mathbf{0 . 0 0 1} & 1.04 & 0.66-1.63 & 0.862 \\ \text { IV } & 1.64 & 0.81-3.31 & 0.172 & 0.86 & 0.57-1.761 & 0.859\end{array}$

Tumor classification (pT)

$\begin{array}{lllllll}\text { pT1-pT2 } & 1.16 & 0.27-5.03 & 0.846 & 0.56 & 0.22-1.41 & 0.218 \\ \text { pT3-pT4 } & 0.61 & 0.40-0.94 & \mathbf{0 . 0 2 4} & 1.03 & 0.75-1.42 & 0.862\end{array}$

Lymph node metastasis $(\mathrm{pN})$

$\begin{array}{lllllll}\text { pN0 } & 0.73 & 0.33-1.62 & 0.436 & 0.77 & 0.43-1.40 & 0.377 \\ \text { pN1-2 } & 0.86 & 0.47-1.60 & 0.640 & 1.04 & 0.73-1.45 & 0.814\end{array}$

Tumor grade (WHO)

$\begin{array}{lllllll}1-2 & 0.73 & 0.35-0.80 & \mathbf{0 . 0 0 3} & 0.93 & 0.6-1.30 & 0.665 \\ 3-4 & 0.89 & 0.30-2.61 & 0.829 & 0.91 & 0.37-2.22 & 0.837\end{array}$

Systemic inflammatory response (CRP)

$\begin{array}{lllllll}\leq 8.7 & 0.53 & 0.35-0.80 & \mathbf{0 . 0 0 2} & 0.92 & 0.59-1.41 & 0.688 \\ >8.7 & 0.95 & 0.45-2.01 & 0.886 & 2.04 & 1.04-4.00 & \mathbf{0 . 0 3 8}\end{array}$

Abbreviations: CI, confidence interval; CRP, C-reactive protein; HR, hazard ratio; TLR, toll-like receptor 
Supplementary Table 4. Survival analysis by subgroups, high TLR5 expression compared to low and positive TLR7 expression compared to negative in 549 colorectal cancer patients

\begin{tabular}{|c|c|c|c|c|c|c|}
\hline & \multicolumn{3}{|c|}{ High TLR5 } & \multicolumn{3}{|c|}{ Positive TLR7 } \\
\hline & HR & $95 \%$ CI & $p$ value & HR & $95 \%$ CI & $p$ value \\
\hline \multicolumn{7}{|l|}{ Age } \\
\hline$<65$ & 0.61 & $0.38-0.99$ & 0.044 & 0.55 & $0.28-1.07$ & 0.078 \\
\hline$\geq 65$ & 0.63 & $0.42-0.94$ & 0.025 & 0.47 & $0.29-0.75$ & 0.002 \\
\hline
\end{tabular}

Gender

$\begin{array}{lllllll}\text { Male } & 0.67 & 0.45-1.02 & 0.063 & 0.63 & 0.36-1.11 & 0.111 \\ \text { Female } & 0.54 & 0.34-0.86 & \mathbf{0 . 0 0 9} & 0.38 & 0.22-0.64 & <\mathbf{0 . 0 0 1}\end{array}$

Location

$\begin{array}{lllllll}\text { Right colon } & 0.66 & 0.36-1.22 & 0.187 & 0.32 & 0.17-0.60 & <\mathbf{0 . 0 0 1} \\ \text { Left colon } & 0.67 & 0.38-1.17 & 0.160 & 0.55 & 0.25-1.23 & 0.146 \\ \text { Rectum } & 0.54 & 0.34-0.86 & \mathbf{0 . 0 0 9} & 0.63 & 0.32-1.26 & 0.195\end{array}$

Tumor stage

$\begin{array}{lllllll}\text { I } & 0.89 & 0.24-3.31 & 0.860 & 0.55 & 0.07-1.43 & 0.576 \\ \text { II } & 0.64 & 0.29-1.42 & 0.272 & 0.52 & 0.12-2.21 & 0.379 \\ \text { III } & 0.78 & 0.49-1.22 & 0.268 & 0.68 & 0.40-1.18 & 0.168 \\ \text { IV } & 1.02 & 0.59-1.76 & 0.956 & 0.70 & 0.37-1.35 & 0.291\end{array}$

Tumor classification (pT)

$\begin{array}{lllllll}\text { pT1-pT2 } & 1.16 & 0.45-3.0 & 0.757 & 0.45 & 0.10-1.95 & 0.284 \\ \text { pT3-pT4 } & 0.67 & 0.48-0.94 & \mathbf{0 . 0 2 0} & 0.59 & 0.39-0.89 & \mathbf{0 . 0 1 2}\end{array}$

Lymph node metastasis $(\mathrm{pN})$

$\begin{array}{lllllll}\text { pN0 } & 0.55 & 0.30-1.01 & 0.054 & 1.24 & 0.30-5.11 & 0.768 \\ \text { pN1-2 } & 0.72 & 0.50-1.04 & 0.083 & 0.68 & 0.44-1.04 & 0.075\end{array}$

Tumor grade (WHO)

$\begin{array}{lllllll}1-2 & 0.59 & 0.41-0.83 & \mathbf{0 . 0 0 3} & 0.53 & 0.33-0.86 & \mathbf{0 . 0 1 0} \\ 3-4 & 0.94 & 0.40-2.21 & 0.881 & 0.55 & 0.21-1.39 & 0.205\end{array}$

Systemic inflammatory response (CRP)

$\begin{array}{lllllll}\leq 8.7 & 0.59 & 0.37-0.92 & \mathbf{0 . 0 2 1} & 0.53 & 0.28-1.00 & \mathbf{0 . 0 4 9} \\ >8.7 & 0.58 & 0.31-1.07 & 0.079 & 3.19 & 0.44-23.1 & 0.251\end{array}$

Abbreviations: CI, confidence interval; CRP, C-reactive protein; HR, hazard ratio; TLR, toll-like receptor 